\title{
Figuras de Atividades Funcionais: Concordância de Nomeação e Familiaridade ${ }^{1}$
}

\author{
Tania Fernandes Campos ${ }^{2}$ \\ Soraya Medeiros de Carvalho \\ Luciana Protásio de Melo \\ Ana Carolina de Azevedo Lima \\ Universidade Federal do Rio Grande do Norte
}

\begin{abstract}
RESUMO - O objetivo do estudo foi verificar a concordância de nomeação e familiaridade de um conjunto de figuras de objetos/animais e de atividades funcionais. Participaram 26 crianças entre 5 e 7 anos e 24 estudantes universitários entre 18 e 25 anos, de ambos os sexos. Os participantes observaram 90 figuras, 45 de atividades funcionais e 45 de objetos/animais, nomearam uma a uma e referiram o grau de familiaridade. Os dados foram analisados pelo coeficiente de Kappa, correlação de Spearman e teste de Mann-Whitney. Os resultados mostraram que a concordância com o nome original foi maior do que a frequiência de outras nomeações. As crianças tiveram mais dificuldades em fazer a nomeação. As figuras de objetos/animais foram consideradas mais familiares. O estudo mostrou diferenças significativas entre as figuras e entre as crianças e adultos, sugerindo o uso de figuras de atividades funcionais em estudos neuropsicológicos.
\end{abstract}

Palavras-chave: figuras de atividades funcionais; nomeação; familiaridade.

\section{Functional Activities Pictures: Name Agreement and Familiarity}

\begin{abstract}
This study was done with the aim to examine the naming agreement and familiarity of a set of objects/animals and functional activities pictures. Twenty six children were used between 5-7 years old and 24 university students between 18-25 years old of both sexes. Participants should watch 90 pictures, being 45 of functional activities and 45 of objects/animals, nominate one by one, moreover, to refer the familiarity degree. Data were analyzed by coefficient of Kappa, Spearman correlation test and Mann-Whitney test. The original name agreement was bigger than the frequency of other nominations. The children had more difficult than adults to make the pictures nomination. The objects/animals pictures were considered more familiar, as well children as adults. This way, the study showed significant differences between the pictures and between children and adults, suggesting the use of functional activities pictures in neuropsychological studies.
\end{abstract}

Key words: functional activities pictures; naming; familiarity.

Estudos mostram que um estímulo visual pode facilitar a eficiência, precisão e velocidade com que o sistema perceptual identifica e processa a informação recebida (Greene, Easton \& Lashell, 2001; Stenberg, Radeborg \& Hedman, 1995). Além disso, figuras são utilizadas, a fim de comparar o efeito dos estímulos visual e verbal e correlacionar com a localização de lesões cerebrais (Allegri, Harris, Serrano \& Delavald, 2001; Yetkin, Rosenberg, Weiner, Purdy \& Cullum, 2006). Também observa-se que estímulos pictoriais possibilitam a compreensão do funcionamento dos processos mnemônicos como a aquisição, codificação e evocação (Degenszajn, Caramelli, Caixeta \& Nitrini, 2001).

Testes com figuras em preto e branco, de objetos e animais, de faces conhecidas ou desconhecidas, são freqüientemente utilizados para avaliação da memória explícita (Ballesteros, Reales, Garcia \& Carrasco, 2006) e implícita (Zago, Fenske, Aminoff \& Martinos, 2005). Figuras de paisagens, pessoas mutiladas e de crianças, por serem reconhecidas como estímulos agradáveis ou desagradáveis, são indicadas para avaliação da memória

1 Agradecimentos: Ao Prof. Alexandre Augusto de Lara Menezes do Dept. de Fisiologia da UFRN, pela colaboração na seleção das figuras, e aos alunos do Curso de Fisioterapia da UFRN, pela contribuição na coleta dos dados.

2 Endereço: Universidade Federal do Rio Grande no Norte, Depto. de Fisioterapia, Av. Senador Salgado Filho, 3000, Natal, RN, Brasil 59066-800. E-mail: taniacampos@ufrnet.br emocional (Abrisqueta-Gomez, Ueta, Oliveira, Bertolucci \& Bueno, 1998; Arntz, De Groot \& Kindt, 2005). Entretanto, a realização desses testes é precedida por um processo de validação das figuras. No Brasil, Pompéia, Miranda e Bueno (2001) validaram, quanto a nomeação, familiaridade e complexidade visual, 400 figuras de animais e objetos, retiradas do estudo original de Snodgrass e Vanderwart (1980), obtendo índices satisfatórios de confiabilidade e validade ao ser comparado com estudos internacionais (Pompéia, Miranda \& Bueno, 2003).

Pesquisas têm mostrado que a utilização de figuras de ações pode ser importante na avaliação neuropsicológica de pacientes com lesões cerebrais, a fim de ser estabelecido o perfil neuropsicológico e a correlação com a localização das lesões, entretanto, resultados são ainda controversos. No estudo com a Doença de Alzheimer, pacientes responderam mais rápido e fizeram menos erros na nomeação de figuras de objetos (substantivos) do que de figuras de ações (verbos) (Druks \& cols., 2006). Por outro lado, estudos têm encontrado que a nomeação de verbos é mais comprometida do que a de substantivos, sugerindo que verbos são semanticamente mais complexos (Kim \& Thompson, 2004; Robinson, Grossman, White-Devine \& D'Esposito, 1996).

Os resultados encontrados vêm indicando que a nomeação de figuras pode ser seletivamente comprometida após lesão cerebral se o córtex ou as conexões envolvidas no processamento 
de um atributo crítico dos conceitos representados pelas figuras são afetados (Lu \& cols., 2002). Segundo Warrington e Shallice (1984), o atributo de figuras representando coisas vivas (animais, frutas, vegetais e flores) é a forma visual e de coisas não-vivas (ferramentas, utensílios de cozinha, móveis e itens de transporte) é a sua função. Outros diferentes atributos podem ser relevantes para a representação de figuras incluindo cor, movimento, faixa de interação e modalidades sensoriais (Lu \& cols., 2002).

Os achados da avaliação diagnóstica estão diretamente relacionados com a programação da intervenção terapêutica. Estudos já relataram que o programa de tratamento deve priorizar as tarefas genéricas no lugar das ações específicas, sendo assim, adequado adotar uma abordagem direcionada para as atividades funcionais, em tarefas e habilidades que possam ser diretamente aplicadas no dia-a-dia (Abrisqueta-Gomez \& cols., 2004; Ávila, 2003). Uma seqüência de movimentos aprendidos pode ser mais fortemente associada com a apresentação de figuras de objetos pessoais familiares usados rotineiramente pelos pacientes com demência do que de objetos não-familiares, o que tem contribuído para o planejamento do tratamento (Giovannetti \& cols., 2006).

$\mathrm{Na}$ literatura, não foram encontrados estudos com figuras específicas de atividades funcionais, como as atividades básicas da vida diária (ABVDs) e as atividades instrumentais de vida diária (AIVDs). As ABVDs dizem respeito aos cuidados pessoais da vida diária, como alimentar-se, vestir-se, fazer higiene e mover-se. As AIVDs são habilidades mais avançadas e consideradas como determinantes para a independência do indivíduo na comunidade, como cozinhar, fazer compras, executar tarefas domésticas, dirigir, entre outras (Almeida \& Nitrini, 1995). Essas atividades são realizadas com bastante freqüência no dia-a-dia e ao longo da história de vida do indivíduo. A familiaridade pré-mórbida com um conceito é considerada um preditor benéfico do desempenho de pacientes com demência semântica (Rogers, Patterson \& Graham, 2007). A análise de efeitos de percepção de categoria semântica na representação interna cerebral demonstrou que verbos produzem mais distinta representação interna por causa de seu envolvimento mais direto com as ações motoras (Cangelosi \& Harnad, 2000).

Pelos dados da literatura, utilizando figuras de ações relacionadas com o desempenho motor, é possível supor que o uso de figuras de atividades funcionais possa determinar a habilidade para coordenar mentalmente, manipular uma diversificada faixa de informações que pode estar associada com a atividade apresentada e contribuir para maior representação interna cerebral. Dessa forma, sentiu-se a necessidade de realizar um estudo com o objetivo de validar um conjunto de figuras de atividades funcionais, quanto à nomeação e à familiaridade, comparando com figuras de objetos/animais, entre crianças e adultos jovens, a fim de que essas figuras possam ser utilizadas em estudos posteriores, visando facilitar a recuperação das habilidades funcionais de pacientes com comprometimento cognitivo-motor.

\section{Amostra}

\section{Método}

Participaram 26 estudantes de uma entidade particular do ensino fundamental entre 5 e 7 anos de idade (oito meninos e 18 meninas) e 24 estudantes de uma entidade do ensino superior entre 18 e 25 anos (cinco homens e 19 mulheres). Foram ex- cluídos os indivíduos que faziam uso de medicação ou tinham alguma patologia. O termo de consentimento foi assinado pelos pais das crianças e pelos estudantes universitários e o estudo recebeu a aprovação do Comitê de Ética em Pesquisa.

\section{Procedimentos}

Foram selecionadas do CorelDRAW noventa figuras coloridas, 45 de atividades funcionais (Figura 1), que representavam ABVDs e AIVDs, e 45 de objetos/animais (Figura 2). As figuras foram nomeadas previamente, impressas em cartões de $7 \times 7 \mathrm{~cm}$, misturadas aleatoriamente e em seguida apresentadas uma de cada vez. Durante a apresentação, os indivíduos faziam a nomeação das figuras (sem saberem a nomeação dada anteriormente) e determinavam o grau de familiaridade (1- pouco familiar; 3 - médio; 5 - muito familiar). Duas sessões de apresentação foram realizadas pelo mesmo examinador num intervalo de dois dias. Foram dadas instruções para que a nomeação das figuras de atividades funcionais fosse realizada com base na ação expressa na figura, para isso questionou-se: O que está acontecendo nessa figura? Que nome você dá para ela? Para avaliar a familiaridade, perguntou-se aos participantes: Quanto essa figura é familiar para você, pouco (1), médio (3) ou muito (5)?

Após a coleta, foram obtidas as seguintes medidas:

a) Concordância de nomeação: percentual de indivíduos que fizeram a nomeação de acordo com o nome original. Para as figuras de atividades funcionais, considerou-se como concordante o nome que representava uma ação correspondente a atividade apresentada na figura, independentemente se o verbo utilizado fosse diferente. Ex: partindo carne $=$ cortando carne. Foi classificado como satisfatório o percentual de concordância acima de $50 \%$.

b) Confiabilidade teste-reteste: índice de correlação entre os dois testes quanto a concordância de nomeação e ao grau de familiaridade.

c) Nome freqüente: nomeação que obteve maior freqüência percentual para os adultos e crianças. Quando houve desacordo, foi realizado um julgamento para estabelecer a nomeação mais adequada.

d) Familiaridade: grau de familiaridade com a ação ou objeto e animal mostrados nas figuras.

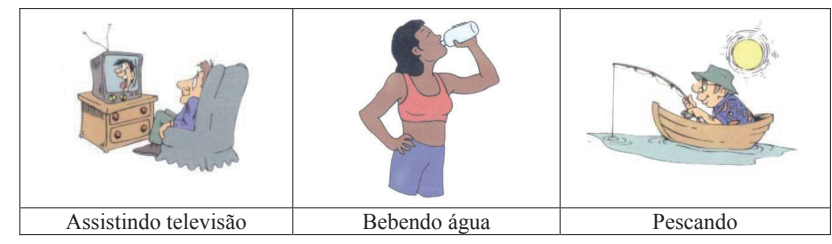

Figura 1. Apresentação de três figuras de atividades funcionais utilizadas no estudo, representando atividades básicas e instrumentais da vida diária (original colorido).

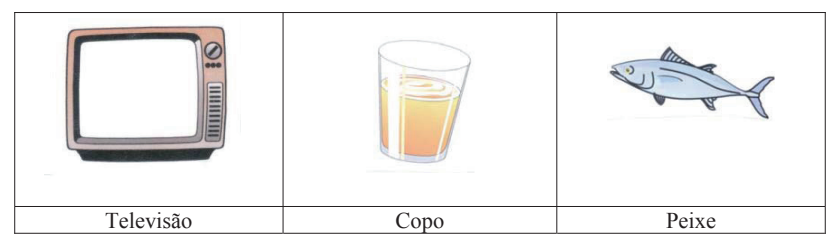

Figura 2. Apresentação de três figuras de objetos/animais utilizadas no estudo (original colorido). 
Todos os procedimentos foram baseados no estudo de Pompéia e cols. (2001), assim como no estudo de Cycowicz, Friedman, Snodgrass e Duff. (2001).

\section{Análise de dados}

Os dados foram analisados pelo programa SPSS 12.00 (Statistical Package for the Social Science), atribuindo-se o nível de significância de 5\%. A comparação da frequiência de concordância entre a nomeação prévia das figuras e a estabelecida pelos participantes foi verificada pelo teste Quiquadrado. A confiabilidade teste-reteste quanto à nomeação foi analisada pelo Índice de Kappa e para a familiaridade pelo teste de correlação de Spearman. As diferenças entre crianças e adultos quanto ao grau de familiaridade foram verificadas pelo teste de Mann-Whitney e quanto ao tipo de figura pelo teste de Wilcoxon.

\section{Resultados}

\section{Concordância de nomeação}

Para as crianças, a frequiência percentual de concordância de nomeação no primeiro teste foi em média $74 \%$ para as figuras de atividades funcionais e $85 \%$ para as figuras de objetos/animais e, no segundo teste, foi de $75 \%$ e $88 \%$, respectivamente. Nos adultos jovens, observou-se no primeiro teste uma concordância de $84 \%$ nas figuras de atividades funcionais e de $97 \%$ nas figuras de objetos/animais e, no segundo teste, foi de $87 \%$ e $99 \%$, respectivamente. No primeiro teste, não foram encontradas diferenças significativas entre crianças e adultos quanto à frequiência de concordância de nomeação nas figuras de atividades funcionais $(p=0,392)$ e de objetos/animais $(p=0,149)$, assim como no segundo teste (atividades funcionais: $p=0,288$ e de objetos/animais: $p=0,619$ ). Para as crianças, não se verificou diferença nos percentuais entre os testes (atividades funcionais: $p=0,936$; objetos/animais: $p=0,758$ ), como também para os adultos (atividades funcionais: $p=0,765$; objetos/animais: $p=0,619$ ).

Concordância maior do que $50 \%$ foi encontrada para a maioria das figuras. Índices igual ou abaixo de 50\% foram encontrados no primeiro teste em três figuras de atividades funcionais para as crianças ("andando de ônibus", "arrancando dente" e "digitando no computador") e em três para os adultos ("andando de ônibus", "arrancando dente" e "cuidando de criança"). Além de cinco figuras de objetos/animas ("aspirador", "barbeador", "churrasqueira", "sinuca" e "tapete") para as crianças e em uma figura para os adultos ("churrasqueira"). No segundo teste, observou-se para as crianças em duas figuras de atividades funcionais ("arrancando dente" e "digitando no computador") e para os adultos nas mesmas figuras do primeiro teste. Para as figuras de objetos/animas, apenas as crianças apresentaram esses índices em três figuras ("aspirador", "churrasqueira" e "sinuca") (Tabelas 1 e 2).

Do total de figuras para os adultos, oito de atividades funcionais e 32 de objetos/animais no primeiro teste e 18 e 41 figuras, respectivamente, no segundo teste, apresentaram $100 \%$ de concordância, ou seja, os nomes atribuídos concordaram com a ação ou o objeto/animal apresentados na figura. Para as crianças, os resultados foram semelhantes entre os dois testes, somente houve concordância de $100 \%$ em 13 de objetos/animais (Tabelas 1 e 2).

\section{Confiabilidade teste-reteste}

O índice de Kappa mostrou correlação significativa da nomeação entre os dois testes em 37 figuras de atividades funcionais e 36 figuras de objetos/animais para as crianças e em 37 figuras de atividades funcionais e para todas as figuras de objetos/animais nos adultos (Tabela 3). Quanto à familiaridade, verificou-se correlação significativa, com os adultos apresentando maiores coeficientes tanto para as figuras de atividades funcionais (criança: $\mathrm{R}=0,31, \mathrm{p}=0,001$; adulto: $\mathrm{R}=0,73, \mathrm{p}=0.001$ ) quanto para as figuras de objetos/animais (criança: $\mathrm{R}=0,51, \mathrm{p}=0,001$; adulto: $\mathrm{R}=0,78, \mathrm{p}=0,001$ ).

\section{Nome freqüente}

Em algumas figuras, foi possível observar nomeações semelhantes às estabelecidas anteriormente, ocorrendo apenas mudança nos verbos, porém estes apresentavam o mesmo significado. A figura "falando ao telefone" foi nomeada mais freqüentemente como "atendendo telefone", assim como "partindo carne" que foi chamada como "cortando carne", "andando de ônibus" ficou "passeando de ônibus", "cuidando de criança" como "segurando o bebê", "tomando mamadeira" foi chamada mais de "dando mamadeira" e "dirigindo moto" como "andando de moto".

Alguns nomes mostraram abreviação em relação aos nomes anteriores, como "digitando no computador", que ficou somente "digitando", "dirigindo carro" como "dirigindo" e "espanando móveis" como "espanando". Por outro lado, nomes pequenos receberam especificações como "plantando" que ficou "plantando flores" e, nas figuras de objetos, "copo" que foi especificada como "copo de suco" e "aspirador" como "aspirador de pó", porém estes últimos nomes não foram os mais freqüentes.

Várias interpretações foram observadas em algumas figuras. A figura "passando o aspirador" recebeu nomeações como "limpando a casa" e "tirando a sujeira", porém o nome mais freqüente foi "limpando o chão" e a figura "arrancando dente" foi chamada de "fazendo uma consulta", mas a nomeação mais freqüente foi "indo ao dentista". Todos os nomes das figuras considerados mais freqüentes encontram-se nas Tabelas 1 e 2.

\section{Familiaridade}

Observou-se diferença entre adultos e crianças quanto ao grau de familiaridade, nas figuras de atividades funcionais $(p<0,001)$ e de objetos/animais $(p<0,01)$, com as crianças apresentando escores maiores do que os adultos em ambos os tipos de figuras. Foi verificado que as figuras de objetos/animais tiveram escore maior do que as figuras de atividades funcionais, tanto para as crianças $(p<0,0001)$ quanto para os adultos $(p=0,01)$.

\section{Discussão}

No estudo, foi encontrada concordância satisfatória de nomeação para os dois tipos de figuras, os dois testes realizados e para ambos os grupos estudados. O índice de Kappa confirmou o nível aceitável de confiabilidade teste-reteste 
Tabela 1. Percentuais de concordância entre a nomeação prévia e a estabelecida por crianças e adultos para as figuras de atividades funcionais no primeiro e segundo testes e nome mais freqüente apresentado por ambos os grupos.

\begin{tabular}{|c|c|c|c|c|c|}
\hline FIGURA & TESTE 1 & & TESTE 2 & & NOME FREQÜENTE \\
\hline & Criança & Adulto & Criança & Adulto & \\
\hline Aguando plantas & 75 & 96 & 76 & 100 & Aguando plantas \\
\hline Andando de cavalo & 62 & 79 & 54 & 63 & Andando de cavalo \\
\hline Andando de bicicleta & 69 & 58 & 58 & 67 & Andando de bicicleta \\
\hline Andando de ônibus & 42 & 17 & 54 & 21 & Passeando de ônibus \\
\hline Apagando fogo & 73 & 92 & 65 & 88 & Apagando fogo \\
\hline Arrancando dente & 27 & 17 & 31 & 17 & Indo ao dentista \\
\hline Assistindo televisão & 89 & 100 & 85 & 100 & Assistindo televisão \\
\hline Bebendo água & 96 & 100 & 85 & 100 & Bebendo água \\
\hline Brincando de boneca & 92 & 96 & 89 & 100 & Brincando de boneca \\
\hline Cantando & 62 & 83 & 73 & 83 & Cantando \\
\hline Cozinhando & 69 & 92 & 76 & 96 & Cozinhando \\
\hline Cuidando de criança & 65 & 42 & 54 & 46 & Segurando o bebê \\
\hline Digitando no computador & 50 & 75 & 42 & 71 & Digitando \\
\hline Dirigindo carro & 58 & 58 & 54 & 63 & Dirigindo \\
\hline Dirigindo moto & 69 & 54 & 73 & 58 & Andando de moto \\
\hline Dormindo & 89 & 100 & 92 & 96 & Dormindo \\
\hline Empinando pipa & 92 & 88 & 92 & 96 & Soltando pipa \\
\hline Enxugando os pratos & 65 & 88 & 77 & 92 & Enxugando os pratos \\
\hline Escovando os dentes & 77 & 100 & 88 & 100 & Escovando os dentes \\
\hline Escrevendo & 65 & 83 & 73 & 96 & Escrevendo \\
\hline Espanando móveis & 73 & 100 & 81 & 100 & Espanando \\
\hline Falando ao telefone & 65 & 83 & 65 & 83 & Atendendo telefone \\
\hline Fazendo barba & 81 & 88 & 89 & 96 & Fazendo a barba \\
\hline Fazendo churrasco & 62 & 71 & 62 & 79 & Fazendo churrasco \\
\hline Furando a parede & 73 & 88 & 77 & 92 & Furando a parede \\
\hline Jogando basquete & 46 & 58 & 58 & 71 & Jogando basquete \\
\hline Jogando cartas & 89 & 88 & 89 & 100 & Jogando cartas \\
\hline Jogando dados & 85 & 88 & 92 & 100 & Jogando dados \\
\hline Jogando sinuca & 46 & 96 & 58 & 100 & Jogando sinuca \\
\hline Lavando as mãos & 73 & 88 & 81 & 96 & Lavando as mãos \\
\hline Lendo um livro & 81 & 96 & 89 & 100 & Lendo um livro \\
\hline Limpando o tapete & 81 & 100 & 85 & 100 & Limpando o tapete \\
\hline Partindo carne & 85 & 88 & 81 & 100 & Cortando carne \\
\hline Passando o aspirador & 69 & 88 & 77 & 88 & Limpando o chão \\
\hline Passando roupas & 89 & 88 & 76 & 92 & Passando roupas \\
\hline Pescando & 73 & 96 & 73 & 100 & Pescando \\
\hline Pintando quadro & 81 & 88 & 85 & 100 & Pintando quadro \\
\hline Plantando & 81 & 88 & 81 & 92 & Plantando flores \\
\hline Pulando corda & 96 & 100 & 92 & 100 & Pulando corda \\
\hline Remando & 77 & 79 & 69 & 96 & Remando \\
\hline Tocando violão & 73 & 96 & 73 & 96 & Tocando violão \\
\hline Tomando banho & 89 & 100 & 96 & 100 & Tomando banho \\
\hline Tomando mamadeira & 85 & 83 & 81 & 92 & Dando mamadeira \\
\hline Tomando sorvete & 85 & 88 & 85 & 100 & Tomando sorvete \\
\hline Varrendo a casa & 89 & 96 & 89 & 100 & Varrendo a casa \\
\hline
\end{tabular}

para a nomeação. As figuras de atividades funcionais foram mais difíceis de serem nomeadas, houve uma freqüência maior de nomes, diferentes verbos foram utilizados e os participantes nomearam com mais detalhes como em "apagando fogo", que foi nomeada como "homem tirando fogo do prédio", e a figura "fazendo churrasco" como "fazendo churrasco de salsicha". Segundo estudo anterior, esse tipo de figura exige mais atenção, percepção, discriminação dos elementos incluídos na ação, bem como de conteúdos da memória de longo-prazo (Szekely \& cols., 2005). Outro aspecto importante de ser analisado é que todas as figuras de ações envolvem ao menos uma pessoa, animal ou objeto, e muitas delas envolvem dois ou mais protagonistas e isso é necessário para produzir significados relacionais, além disso, existe uma 
Figuras de Atividades Funcionais

Tabela 2. Percentuais de concordância entre a nomeação prévia e a estabelecida por crianças e adultos para as figuras de objetos/animais no primeiro e segundo testes e nome mais freqüente apresentado por ambos os grupos.

\begin{tabular}{|c|c|c|c|c|c|}
\hline FIGURA & TESTE 1 & & TESTE 2 & & NOME FREQÜENTE \\
\hline & Criança & Adulto & Criança & Adulto & \\
\hline Aspirador & 46 & 100 & 50 & 100 & Aspirador \\
\hline Barbeador & 50 & 100 & 69 & 100 & Barbeador \\
\hline Barco & 96 & 100 & 96 & 100 & Barco \\
\hline Bicicleta & 100 & 100 & 100 & 100 & Bicicleta \\
\hline Bola de basquete & 81 & 92 & 77 & 100 & Bola de basquete \\
\hline Boneca & 89 & 96 & 96 & 100 & Boneca \\
\hline Cama & 100 & 100 & 100 & 100 & Cama \\
\hline Carne & 81 & 100 & 85 & 100 & Carne \\
\hline Carro & 100 & 100 & 100 & 100 & Carro \\
\hline Carro de bombeiro & 65 & 100 & 73 & 100 & Carro de bombeiro \\
\hline Cartas & 92 & 88 & 100 & 100 & Cartas \\
\hline Cavalo & 100 & 100 & 100 & 100 & Cavalo \\
\hline Churrasqueira & 35 & 50 & 35 & 71 & Churrasqueira \\
\hline Chuveiro & 96 & 100 & 100 & 100 & Chuveiro \\
\hline Computador & 100 & 100 & 96 & 100 & Computador \\
\hline Copo & 73 & 75 & 69 & 75 & Copo \\
\hline Corda & 61 & 96 & 65 & 100 & Corda \\
\hline Criança & 96 & 83 & 81 & 92 & Criança \\
\hline Dados & 96 & 100 & 96 & 100 & Dados \\
\hline Dente & 81 & 100 & 81 & 100 & Dente \\
\hline Escova de dente & 96 & 100 & 100 & 100 & Escova de dente \\
\hline Ferro & 77 & 100 & 92 & 100 & Ferro \\
\hline Flor & 100 & 100 & 100 & 100 & Flor \\
\hline Fogão & 89 & 100 & 89 & 100 & Fogão \\
\hline Furadeira & 89 & 96 & 81 & 100 & Furadeira \\
\hline Lápis & 100 & 100 & 100 & 100 & Lápis \\
\hline Livro & 96 & 100 & 100 & 100 & Livro \\
\hline Mamadeira & 100 & 100 & 100 & 100 & Mamadeira \\
\hline Mangueira & 81 & 100 & 77 & 100 & Mangueira \\
\hline Mãos & 96 & 100 & 100 & 100 & Mãos \\
\hline Microfone & 77 & 100 & 81 & 100 & Microfone \\
\hline Moto & 100 & 100 & 100 & 100 & Moto \\
\hline Móveis & 89 & 100 & 85 & 100 & Móveis \\
\hline Ônibus & 89 & 96 & 92 & 100 & Ônibus \\
\hline Peixe & 100 & 100 & 100 & 100 & Peixe \\
\hline Pincel & 85 & 100 & 92 & 100 & Pincel \\
\hline Pipa & 100 & 100 & 100 & 100 & Pipa \\
\hline Prato & 92 & 96 & 92 & 100 & Prato \\
\hline Sinuca & 23 & 83 & 50 & 100 & Sinuca \\
\hline Sorvete & 85 & 96 & 92 & 96 & Sorvete \\
\hline Tapete & $\mathbf{5 0}$ & 100 & 73 & 100 & Tapete \\
\hline Telefone & 100 & 100 & 100 & 100 & Telefone \\
\hline Televisão & 96 & 100 & 100 & 100 & Televisão \\
\hline Vassoura & 100 & 96 & 96 & 100 & Vassoura \\
\hline Violão & 96 & 100 & 96 & 100 & Violão \\
\hline
\end{tabular}

dificuldade em categorizar semanticamente figuras de ações (Vigliocco, Vinson, Damian \& Levelt, 2002).

Ficou bem evidente, no presente estudo, que as figuras de atividades funcionais representam contextos de vida, visto que "pulando corda" foi dito "brincando"; "limpando tapete" como "fazendo faxina"; "digitando" como "entrando na internet" e "fazendo tarefa"; "andando de bicicleta" como "andando de Caloi". Algumas figuras apresentam componente emocional como a figura "arrancando dente" que foi dito "medo de ir ao dentista", a figura "tomando sorvete" foi nomeada "mulher chupando sorvete e homem com inveja dela". Também pode-se observar que os participantes fizeram uma interpretação própria da figura, "passando aspirador" como "fazendo limpeza mais fácil", "escrevendo" como 
T. F. Campos \& cols.

Tabela 3. Índices de concordância de Kappa (p valor) quanto a nomeação entre o primeiro e segundo testes, para as figuras de atividades funcionais e de objetos/animais, encontrados em crianças e adultos.

\begin{tabular}{|c|c|c|c|c|c|}
\hline \multirow{2}{*}{$\begin{array}{l}\text { FIGURA } \\
\text { Atividades funcionais }\end{array}$} & \multicolumn{2}{|c|}{ KAPPA } & \multirow{2}{*}{$\begin{array}{r}\text { FIGURA } \\
\text { Objetos/animais }\end{array}$} & \multicolumn{2}{|c|}{ KAPPA } \\
\hline & Criança & Adulto & & Criança & Adulto \\
\hline Jogando basquete & $0.62(0.01)$ & $0.73(0.01)$ & Bola de basquete & $0.20(0.32)$ & $0.62(0.01)$ \\
\hline Falando ao telefone & $0.66(0.01)$ & $0.40(0.05)$ & Telefone & $\mathrm{a}$ & a \\
\hline Lendo um livro & $0.71(0.01)$ & a & Livro & $0.65(0.01)$ & a \\
\hline Digitando no computador & $0.54(0.01)$ & $0.26(0.19)$ & Computador & $\mathrm{a}$ & $\mathrm{a}$ \\
\hline Dirigindo carro & $0.46(0.02)$ & $0.74(0.01)$ & Carro & a & $\mathrm{a}$ \\
\hline Cantando & $0.57(0.01)$ & $0.70(0.01)$ & Microfone & $0.20(032)$ & a \\
\hline Andando à cavalo & $0.37(0.05)$ & $0.02(0.90)$ & Cavalo & $\mathrm{a}$ & $\mathrm{a}$ \\
\hline Dormindo & $0.34(0.08)$ & a & Cama & a & a \\
\hline Escrevendo & $0.64(0.01)$ & $0.36(0.02)$ & Lápis & $\mathrm{a}$ & $\mathrm{a}$ \\
\hline Tomando banho & $-0.10(0.60)$ & $\mathrm{a}$ & Chuveiro & a & $\mathrm{a}$ \\
\hline Escovando os dentes & $0.08(0.65)$ & a & Escova de dente & a & $\mathrm{a}$ \\
\hline Apagando fogo & $0.64(0.01)$ & $0.78(0.01)$ & Carro de bombeiro & $0.64(0.01)$ & $\mathrm{a}$ \\
\hline Pescando & $0.80(0.01)$ & a & Peixe & $\mathrm{a}$ & a \\
\hline Bebendo água & $0.36(0.02)$ & $\mathrm{a}$ & Copo & $0.34(0.08)$ & $0.56(0.01)$ \\
\hline Partindo carne & $0.60(0.01)$ & $\mathrm{a}$ & Carne & $0.87(0.01)$ & $\mathrm{a}$ \\
\hline Cozinhando & $0.61(0.01)$ & $0.65(0.01)$ & Fogão & $1.00(0.01)$ & a \\
\hline Aguando plantas & $0.51(0.01)$ & $\mathrm{a}$ & Mangueira & $0.65(0.01)$ & $\mathrm{a}$ \\
\hline Passando roupas & $0.34(0.05)$ & $0.78(0.01)$ & Ferro & $0.15(0.35)$ & $\mathrm{a}$ \\
\hline Andando de bicicleta & $0.76(0.01)$ & $0.47(0.02)$ & Bicicleta & $\mathrm{a}$ & $\mathrm{a}$ \\
\hline Jogando sinuca & $0.47(0.01)$ & $\mathrm{a}$ & Sinuca & $0.46(0.01)$ & $\mathrm{a}$ \\
\hline Empinando pipa & $0.34(0.08)$ & $-0.06(0.76)$ & Pipa & a & a \\
\hline Brincando de boneca & $0.78(0.01)$ & $\mathrm{a}$ & Boneca & $-0.06(0.71)$ & $\mathrm{a}$ \\
\hline Pulando corda & $0.65(0.01)$ & $\mathrm{a}$ & Corda & $0.92(0.01)$ & $\mathrm{a}$ \\
\hline Tomando sorvete & $0.41(0.04)$ & $\mathrm{a}$ & Sorvete & $0.63(0.01)$ & $1.00(0.01)$ \\
\hline Fazendo churrasco & $0.51(0.01)$ & $0.56(0.01)$ & Churrasqueira & $0.66(0.01)$ & $0.42(0.02)$ \\
\hline Jogando cartas & $0.62(0.01)$ & $\mathrm{a}$ & Cartas & $\mathrm{a}$ & $\mathrm{a}$ \\
\hline Jogando dados & $0.63(0.01)$ & $\mathrm{a}$ & Dados & $-0.04(0.84)$ & $\mathrm{a}$ \\
\hline Pintando quadro & $0.60(0.01)$ & $\mathrm{a}$ & Pincel & $0.63(0.01)$ & $\mathrm{a}$ \\
\hline Lavando as mãos & $0.57(0.01)$ & $0.47(0.01)$ & Mãos & a & a \\
\hline Tocando violão & $0.78(0.01)$ & $-0.04(0.83)$ & Violão & $1.00(0.01)$ & $\mathrm{a}$ \\
\hline Plantando & $0.51(0.01)$ & $0.45(0.03)$ & Flor & $\mathrm{a}$ & $\mathrm{a}$ \\
\hline Fazendo barba & $0.71(0.01)$ & $0.65(0.01)$ & Barbeador & $0.62(0.01)$ & a \\
\hline Varrendo a casa & $0.62(0.01)$ & $\mathrm{a}$ & Vassoura & $\mathrm{a}$ & $\mathrm{a}$ \\
\hline Passando o aspirador & $0.61(0.01)$ & $0.24(0.24)$ & Aspirador & $0.31(0.13)$ & a \\
\hline Remando & $0.81(0.01)$ & $0.28(0.05)$ & Barco & $-0.04(0.84)$ & a \\
\hline Enxugando pratos & $0.17(0.37)$ & $1.00(0.01)$ & Prato & $-0.08(0.67)$ & $\mathrm{a}$ \\
\hline Limpando tapete & $0.87(0.01)$ & a & Tapete & $0.39(0.03)$ & a \\
\hline Espanando móveis & $0.42(0.03)$ & $\mathrm{a}$ & Móveis & $0.51(0.01)$ & $\mathrm{a}$ \\
\hline Arrancando dente & $0.53(0.01)$ & $0.40(0.05)$ & Dente & $1.00(0.01)$ & a \\
\hline Furando a parede & $0.78(0.01)$ & $-0.11(0.58)$ & Furadeira & $0.71(0.01)$ & a \\
\hline Andando de ônibus & $0.16(0.39)$ & $0.59(0.01)$ & Ônibus & $0.78(0.01)$ & a \\
\hline Cuidando de criança & $0.76(0.01)$ & $0.58(0.01)$ & Criança & $0.29(0.04)$ & $0.62(0.01)$ \\
\hline Tomando mamadeira & $0.87(0.01)$ & $0.25(0.19)$ & Mamadeira & $\mathrm{a}$ & $\mathrm{a}$ \\
\hline Dirigindo moto & $0.72(0.01)$ & $0.58(0.01)$ & Moto & a & $\mathrm{a}$ \\
\hline Assistindo televisão & $0.84(0.01)$ & $\mathrm{a}$ & Televisão & $\mathrm{a}$ & $\mathrm{a}$ \\
\hline
\end{tabular}

a: Indica concordância de $100 \%$.

"corrigindo prova" ou "fazendo as contas", "cozinhando" como "fazendo sopa". Esses resultados mostram a possibilidade de explorar figuras de atividades funcionais para a compreensão de diferentes situações de vida e utilização em testes neuropsicológicos. No estudo de Greene e cols. (2001), verificou-se que uma figura de ação facilita tanto o reconhecimento visual, quanto auditivo, por exemplo, a figura de um bebê chorando pode ser identificada de forma visual e auditiva. No estudo de Okochi, Takahashi, Takamuku, Matsuda e Takagi (2005), observou-se que figuras de ações facilitaram o entendimento do idoso a determinar o grau de comprometimento da capacidade funcional.

As figuras de objetos e ações invariavelmente diferem em suas propriedades lexicais (substantivos e verbos, respecti- 
vamente) (Szekely \& cols., 2005), além disso, já existem evidências de uma diferenciação na organização cerebral. Figuras de ações causam maior ativação bilateral nas áreas motoras e próximas à região temporal do que as figuras de objetos (Kable, Kan, Wilson, Thompson-Schill \& Chatterjee, 2005). O córtex occipito-temporal lateral contém regiões neurais que processam diferentes aspectos do movimento, no que se refere à percepção de objetos se movendo no espaço e ao conceito de movimento implicado na ação verbal, nesse sentido, coordena as informações perceptual e conceitual das ações e o córtex occipito-temporal ventral está relacionado com a percepção dos objetos (Kable, Lease-Spellmeyer \& Chatterjee, 2002).

No presente estudo, as crianças apresentaram maior número de nomes alternativos nas figuras de atividades funcionais, coincidindo com os achados de Pompéia e cols. (2001), em que sugerem que as crianças têm dificuldade em integrar adequadamente a informação perceptual e conceitual em função de diferenças na capacidade atencional e de discriminação (Cycowicz \& cols., 2001). No entanto, os percentuais de concordância encontrados indicam que as figuras selecionadas representam conceitos que são conhecidos por estudantes universitários e crianças. A literatura mostra que a idade de aquisição é um importante preditor de recuperação de nomes em adultos normais. Observou-se que substantivos (nomes de objetos comuns) são adquiridos cedo no desenvolvimento, enquanto verbos principais (nomes de ações comuns) são raros até o vocabulário expandir para aproximadamente 200 palavras, em função de que estruturas semânticas dos verbos são inerentemente mais complexas. A aquisição de verbos e adjetivos não pode proceder até a criança ter adquirido substantivos suficientes para realizar a predicação (O’Grady, 1987). Outro aspecto importante é que objetos comuns são relativamente fáceis de serem representados em desenhos, ao contrário de ações que freqüentemente são representadas em desenhos estáticos (Davidoff \& Masterson, 1996).

Quanto à familiaridade, a confiabilidade teste-reteste foi maior para os adultos, entretanto, os dois tipos de figuras foram considerados mais familiares pelas crianças. É possível que o caráter lúdico, assim como a cor das figuras, tenha tido influência. Considera-se que representações de objetos são mediadas somente pela forma e detalhes da superfície, entretanto, quando as figuras têm formas similares, a cor é que permite diferenciá-las, mostrando que o reconhecimento de objetos do dia-a-dia é facilitado pela presença da informação da cor, sendo parte integrante da representação do objeto juntamente com a forma e textura (Rossion \& Pourtois, 2004). Apesar desses aspectos, a análise da familiaridade é uma função difícil, pois familiaridade é definida como um julgamento subjetivo de quanto o indivíduo freqüentemente teve contato ou pensa sobre um certo objeto ou conceito (Allegri \& cols., 2001).

Todas as comparações levam à conclusão de que a nomeação de figuras de atividades funcionais difere das figuras de objetos/animais e de acordo com a idade. Elas são mais difíceis de serem nomeadas, apresentam maior número de nomeações e são consideradas menos familiares do que as figuras de objetos/animais. As crianças apresentam mais dificuldades em fazer nomeação, porém consideram essas figuras mais familiares do que os adultos. É necessário ainda avaliar se as figuras de atividades funcionais que representam as ABVDs e AIVDs podem servir como um instrumento para pesquisa cognitiva, elaboração de testes de memória e aplicação clínica.

\section{Referências}

Abrisqueta-Gomez, J., Ueta R. A., Oliveira, M. G. M., Bertolucci, P. H. F. \& Bueno, O. F. A. (1998). Memória de figuras com conteúdo emocional em pacientes com doença de Alzheimer. Revista de Psiquiatria Clínica, 25(2), 84-87.

Allegri, R. F., Harris, P., Serrano, C. \& Delavald, N. (2001). Perfis diferenciais de perda de memória entre a demência frontotemporal e a do tipo Alzheimer. Psicologia: Reflexão e Crítica, 14(2), 317-324.

Almeida, O. P. \& Nitrini, R. (1995). Demência. São Paulo: Fundo Editorial BYK.

Arntz, A., De Groot, C. \& Kindt, M. (2005). Emotional memory is perceptual. Journal of Behavior Therapy and Experimental Psychiatry, 36(1), 19-34.

Ávila, R. (2003). Resultados da reabilitação neuropsicológica em paciente com doença de Alzheimer leve. Revista de Psiquiatria Clínica, 30(4), 139-146.

Ballesteros, S., Reales, J. M., Garcia, E. \& Carrasco, M. (2006). Selective attention affects implicit and explicit memory for familiar pictures at different delay conditions. Psicothema, 18(1), 88-99.

Cangelosi, A. \& Harnad, S. (2000). The adaptive advantage of symbolic theft over sensorimotor toil: Grounding language in perceptual categories. Evolution of Communication, 4(1), 117-142.

Cycowicz, Y. M., Friedman, D., Snodgrass, J. G. \& Duff, M. (2001). Recognition and source memory for pictures in children and adults. Neuropsychologia, 39(3), 255-267.

Davidoff, J. \& Masterson, J. (1996). The development of picture naming: differences between nouns and verbs. Journal of Neurolinguistics, 9(2), 69-84.

Degenszajn, J., Caramelli, P., Caixeta, L. \& Nitrini, R. (2001). Encoding process in delayed recall impairment and rate of forgetting in Alzheimer's disease. Arquivos de Neuropsiquiatria, 59(2A), 171-174.

Druks, J., Masterson, J., Kopelman, M., Clare, L., Rose, A. \& Rai, G. (2006). Is action naming better preserved (than object naming) in Alzheimer's disease and why should we ask? Brain and Language 98, 332-340.

Giovannetti, T., Sestito, N., Libon, D. J., Schmidt, K. S., Gallo, J. L., Gambino, M. \& Chrysikou, E. G. (2006). The influence of personal familiarity on object naming, knowledge, and use in dementia. Archives of Clinical Neuropsychology, 21, 607-614.

Greene, A. J., Easton, G. R. D. \& LaShell, L. S. R. (2001). VisualAuditory events: cross-modal perceptual priming and recognition memory. Consciousness \& Cognition, 10, 425-435.

Kable, J. W., Kan, I. P., Wilson, A., Thompson-Schill, S. L. \& Chatterjee, A. (2005). Conceptual representations of action in the lateral temporal cortex. Journal of Cognitive Neuroscience, 17, 1855-1870.

Kable, J. W., Lease-Spellmeyer, J. \& Chatterjee, A. J. (2002). Neural substrates of action event knowledge. Journal of Cognitive Neuroscience, 14(5), 795-805.

Kim, M. \& Thompson, C. K. (2004). Verb deficits in Alzheimer's disease and agrammatism: Implications for lexical organization. Brain and Language, 88, 1-20.

Lu, L. H., Crosson, B., Nadeau, S. E., Heilman, K. M., GonzalezRothi, L. J., Raymerd, A., Gilmore, R. L., Bauer, R. M. \& Roper, 
S. N. (2002). Category-specific naming deficits for objects and actions: semantic attribute and grammatical role hypotheses. Neuropsychologia, 40, 1608-1621.

O'Grady, W. (1987). Principles of grammar \& learning. Chicago: University of Chicago Press.

Okochi, J., Takahashi, T., Takamuku, K., Matsuda, S. \& Takagi, Y. (2005). Reliability of a geriatric assessment instrument with illustrations. Geriatrics and Gerontology International, 5, 37-47.

Pompéia, S., Miranda, M. C. \& Bueno, O. F. A. (2001). A set of 400 pictures standardized for portuguese: norms for name agreement, familiarity and visual complexity for children and adults. Arquivos de Neuropsiquiatria, 59(2B), 330-337.

Pompéia, S., Miranda, M. C. \& Bueno, O. F. A. (2003). Brazilian standardized norms for a set of pictures are comparable with those obtained internationally. Arquivos de Neuropsiquiatria, 61(4), 916-919.

Robinson, K. M., Grossman, M., White-Devine, T. \& D’Esposito, M. (1996). Category-specific difficulty naming with verbs in Alzheimer's disease. Neurology, 47, 178-182.

Rogers, T. T., Patterson, K. \& Graham, K. (2007). Colour knowledge in semantic dementia: It is not all black and white. Neuropsychologia, 45, 3285-3298.

Rossion, B. \& Pourtois, G. (2004). Revisiting Snodgrass and Vanderwart's object pictorial set: the role of surface detail in basic-level object recognition. Perception, 33, 217-236.

Snodgrass, J. G. \& Vanderwart, M. (1980). A standardized set of 200 pictures: norms for name agreement, image agreement, familiarity, and visual complexity. Journal of Experimental Psychology: Human Learning \& Memory, 6, 174-215.

Stenberg, G. S., Radeborg, K. \& Hedman, L. R. (1995). The picture superiority effect in implicit conceptual memory tests. Journal of Experimental Psychology. Learning, Memory, and Cognition, 23, 425-441.

Szekely, A., D’Amico, S., Devescovi, A., Federmeier, K., Herron, D., Iyer, G., Jacobsen, T., Arevalo, A. L., Vargha, A. \& Bates, E. (2005). Timed action and object naming. Cortex, 41(1), 7-25.

Vigliocco, G., Vinson, D. P., Damian, M. F. \& Levelt, W. (2002). Semantic distance effects on object and action naming. Cognition, 85(B), 61-69.

Warrington, E. K. \& Shallice, T. (1984). Category-specific semantic impairments. Brain, 107, 829-854.

Yetkin, F. Z., Rosenberg, R. N., Weiner, M. F., Purdy, P. D. \& Cullum, C. M. (2006). FMRI of working memory in patients with mild cognitive impairment and probable Alzheimer's disease. European Radiology, 16(1), 193-206.

Zago, L., Fenske, M. J., Aminoff, E. \& Martinos, M. B. (2005). The rise and fall of priming: how visual exposure shapes cortical representations of objects. Cerebral Cortex, 15(11), 1655-1665.

Recebido em 25.09.2006

Primeira decisão editorial em 22.08.2007

Versão final em 05.03.2008

Aceito em 25.08.2008 